\title{
Molecular Cloning, Bioinformatic Analysis, and Expression of Bombyx mori Lebocin 5 Gene Related to Beauveria bassiana Infection
}

\author{
Dingding Lü, ${ }^{1,2}$ Chengxiang Hou, ${ }^{1}$ Guangxing Qin, ${ }^{1}$ Kun Gao, ${ }^{1}$ Tian Chen, ${ }^{1}$ and Xijie Guo ${ }^{1}$ \\ ${ }^{1}$ School of Biotechnology and Sericultural Research Institute, Jiangsu University of Science and Technology, Zhenjiang 212018, China \\ ${ }^{2}$ Nursing School, Zhenjiang College, Zhenjiang 212003, China \\ Correspondence should be addressed to Xijie Guo; guoxijie@126.com
}

Received 4 October 2016; Revised 21 November 2016; Accepted 19 December 2016; Published 17 January 2017

Academic Editor: Qiang Gao

Copyright (C) 2017 Dingding Lü et al. This is an open access article distributed under the Creative Commons Attribution License, which permits unrestricted use, distribution, and reproduction in any medium, provided the original work is properly cited.

\begin{abstract}
A full-length cDNA of lebocin 5 (BmLeb5) was first cloned from silkworm, Bombyx mori, by rapid amplification of cDNA ends. The BmLeb5 gene is 808 bp in length and the open reading frame encodes a 179-amino acid hydroxyproline-rich peptide. Bioinformatic analysis results showed that BmLeb5 owns an O-glycosylation site and four RXXR motifs as other lebocins. Sequence similarity and phylogenic analysis results indicated that lebocins form a multiple gene family in silkworm as cecropins. Quantitative real-time PCR analysis revealed that BmLeb5 was highest expressed in the fat body. In the silkworm larvae infected by Beauveria bassiana, the expression level of BmLeb5 was upregulated in the fat body and hemolymph which are the most important immune tissues in silkworm. The recombinant protein of BmLeb5 was for the first time successfully expressed with prokaryotic expression system and purified. There are no reports so far that the expression of lebocins could be induced by entomopathogenic fungus. Our study suggested that BmLeb5 might play an important role in the immune response of silkworm to defend B. bassiana infection. The results also provided helpful information for further studying the lebocin family functioned in antifungal immune response in the silkworm.
\end{abstract}

\section{Introduction}

Silkworm (Bombyx mori) is one of the important economic insects spinning cocoon. But the silkworm always suffers from diseases in the sericulture production, especially white muscardine caused by infection with entomopathogenic fungi. Beauveria bassiana was the first discovered fungus causing the devastating white muscardine disease to the silkworms and is always a troublesome pathogenic fungus in sericulture [1-3]. B. bassiana works by direct penetrating into the insect cuticle and colonize in vivo [4]. Then the fungi fight with the host's innate immune defense, consisting mainly of cellular and humoral mechanisms $[5,6]$. A set of antimicrobial peptides (AMPs) are produced to protect the host against invading microbes in humoral immune system [7]. Insect AMPs are mainly synthesized in the fat body, secreted into the hemolymph, and regulated by Rel family transcription factors via activating the Toll and IMD signaling pathways [8-12].
In the silkworm, the AMPs have been identified into six groups: cecropins [13-15], moricins [16], gloverins [17], attacins [18], enbocins [19], and lebocins [20, 21]. Lebocins are first isolated from silkworm and classified as proline-rich AMPs [20, 22]. Then lebocins also have been identified in Trichoplusia ni, Samia cynthia ricini, Pseudoplusia includes, and Manduca sexta [23-26]. Lebocins are generally synthesized in the form of precursors and usually inactive and need to be proteolytically processed at RXXR motifs to generate short active peptides [27]. Unlike the fact that $M$. sexta active lebocin peptides are located at the N-terminal ends, B. mori lebocins are close to the $\mathrm{C}$-terminal with 32 residue peptides. These peptides are O-glycosylated at $\mathrm{Thr}^{15}$ which is important for the antibacterial activity [22]. But abaecin of the honeybee, whose primary structure and antimicrobial activity resemble lebocins, is not $\mathrm{O}$-glycosylated [28].

Four lebocins 1-4 were isolated from silkworm larvae immunized with Escherichia coli. Lebocins 1 and 2 have the same amino acid sequences and consequently named 
lebocin $1 / 2$. Lebocin $1 / 2$ occurred tissues-specifically in the fat body of silkworm after bacterial induction [20]. Lebocins 3 and 4 could be induced by lipopolysaccharide (LPS) and represent tissues-specifically in the fat body and hemocytes [21]. Lebocin 1-3 showed lower antimicrobial activity against Gram-negative bacteria than cecropin B (an AMP of silkworm) in physiological condition. But they exhibited a higher bactericidal activity against $E$. coli under low-ionic-strength [22]. The self-defense mechanism of these lebocins was not clear. Lebocin 3 was confirmed that it has a synergistic effect with cecropin D in antibacterial activity [29]. Meanwhile, there are reports that silkworm lebocins could be induced by LPS or E. coli, but there are no reports that fungus could induce their expression and they could also not be expressed in vitro so far.

In this study, a new upregulated homologous sequences gene of lebocins was derived from our transcriptome analysis to compare differently expressed genes in the silkworm between $B$. bassiana-infected and normal larvae [30]. In order to elucidate the expression mechanisms of lebocin, the full-length cDNA of BmLeb5 by RACE according to the homologous sequences was cloned. Quantitative realtime PCR was used to analyze the expression characteristics of BmLeb5 in the whole larvae, in the fat body, and in hemolymph at different periods after $B$. bassiana infection. The recombinant protein of BmLeb5 was successfully expressed with prokaryotic expression system and purified.

\section{Materials and Methods}

2.1. Silkworm and Fungal Strains. In this study, the experimental silkworm larvae were p50 strain (provided by Sericultural Research Institute of Chinese Academy of Agricultural Sciences) which were fed with fresh mulberry leaves under a photoperiod of $12 \mathrm{~h}$ light and $12 \mathrm{~h}$ dark at $25^{\circ} \mathrm{C}$. The larvae were raised to third or fifth instar for subsequent experiments. Strain BbHN6 of B. bassiana, originally isolated from the cadaver of muscardine silkworm, was also provided by Sericultural Research Institute of Chinese Academy of Agricultural Sciences as we reported before [31]. The conidia of $B$. bassiana were gathered by culturing the fungus on potato dextrose agar (PDA) for 14 days at $25 \pm 1^{\circ} \mathrm{C}$.

2.2. B. bassiana Inoculation. The concentration of conidia of BbHN6 was adjusted to $1 \times 10^{8}$ conidia $\mathrm{mL}^{-1}$ in distilled water containing $0.01 \%$ ( $\mathrm{vol} \mathrm{vol}^{-1}$ ) Tween-20 after getting rid of mycelia. About 100 larvae of third or 300 larvae of fifth instar silkworm larvae were immersed in conidia suspension for $20 \mathrm{~s}$ and distributed into groups in a relative humidity of breeding environment for 48 hours (hrs) and afterward at ambient humidity. The larvae of control groups were treated in distilled water containing $0.01 \%\left(\mathrm{vol} \mathrm{vol}^{-1}\right)$ Tween-20 and placed in the same breeding environment as the BbHN6infected groups.

2.3. Tissues Collection. The tissues (cuticle, fat body, hemolymph, Malpighian tubule, midgut, and silk gland) of the fifth instar silkworm were collected from both control and BbHN6-infected groups from 8 hours after inoculation (hpi) to $54 \mathrm{hpi}$ at intervals of four hours. The anatomical larvae tissues were quickly put into distilled water containing $0.15 \mathrm{M}$ $\mathrm{NaCl}$ to remove attached mulberry leaf pieces. Then the same tissues of 10 larvae were pooled in a storage tube with $0.5 \mathrm{~mL}$ Trizol reagent (TaKaRa) and immediately frozen in liquid nitrogen. But the hemolymph was directly inpoured into the storage tubes from a ruptured proleg. All the samples were stored at $-80^{\circ} \mathrm{C}$ for the following study. There were three repeated samples for every treatment.

2.4. Cloning of BmLeb5 Gene. The extraction of total RNA was performed from third instar silkworm larvae using Trizol reagent $(\mathrm{TaKaRa})$ and detected for the ratio of $28 \mathrm{~S}: 18 \mathrm{~S}$ by agarose electrophoresis. The open reading frame (ORF) was cloned from the third instar larvae by RT-PCR using the primers Leb5orfF (ATGTACAAGTTTTTAGTATTCAGT) and Leb5orfR (CTATTCTTGGAAAATATCCCTCGG) according to our transcriptome analysis and its homologous gene Lebocin1/2 (NP_001037468.1). Full-length cDNA sequences of BmLeb5 were obtained using SMARTer ${ }^{\circledR}$ RACE $5^{\prime} / 3^{\prime}$ Kit (Clontech) according to the instructions of the manufacturer. The specific $5^{\prime} \mathrm{RACE}$ and $3^{\prime} \mathrm{RACE}$ primers were designed according to ORF of BmLeb5. GSP1 (GATTACGCCAAGCTTGGTCCCTTGTGTTACGGTGGCTCT) was used for cloning the $5^{\prime}$-untranslated regions (UTR). GSP2 (GATTACGCCAAGCTTAGAGCCACCGTAACACAAGGGACC) was used to amplify the $3^{\prime}$-UTR. The PCR program was conducted using the following parameters: denaturation at $94^{\circ} \mathrm{C}$ for $5 \mathrm{~min}$, followed by 25 cycles of $94^{\circ} \mathrm{C}$ for $30 \mathrm{~s}, 68^{\circ} \mathrm{C}$ for $30 \mathrm{~s}, 72^{\circ} \mathrm{C}$ for $3 \mathrm{~min}$, and then a final extension at $72^{\circ} \mathrm{C}$ for $7 \mathrm{~min}$. The nested primers NGSP1 (CGGCTGGATGAACCTCTGGCACG) and NGSP2 (ACATGCGTCGGAGGATCAAGAAG) were used for generating good RACE products of $5^{\prime}$ - and $3^{\prime}$-UTR using the same PCR programs.

The DNA products were gel-purified according to the instructions of the kit. Then purified DNA was cloned into pMD19-T vector (TaKaRa) and transformed into E. coli Top 10. The positive clones were identified by PCR and sequencing. The sequences of BmLeb5 were assembled with the obtained fragments from $5^{\prime} \mathrm{RACE}$ and $3^{\prime} \mathrm{RACE}$ using DNASTAR software and analyzed using the National Center for Biotechnology Information (NCBI) BLAST algorithm.

2.5. Bioinformatic Analysis of BmLeb5. BmLeb5 sequence obtained was analyzed using the SeqBuilder program in DNASTAR software package. The signal peptide was predicted with SignalP 4.1 (http://www.cbs.dtu.dk/services/ SignalP/). The potential O-glycosylation sites were predicted with NetOGlyc 4.0 (http://www.cbs.dtu.dk/services/ NetOGlyc/). The homologous sequences were found by BLASTp (https://blast.ncbi.nlm.nih.gov/Blast.cgi). Multiple protein sequences were aligned using Clustalx 2.1 software. The phylogenetic tree was constructed in MEGA 7 [32] using neighbor-joining method [33].

2.6. Quantitative Real-Time PCR ( $q R T-P C R$ ). The relative expression levels of BmLeb5 were detected between control and BbHN6-infected silkworm using qRT-PCR. Total RNAs 
were extracted, respectively, from third instar whole larvae of both the control and the BbHN6-infected groups at 9, 12, $15,18,21,24,27,30,33,36,39,42$, and 45 hpi by using a RNApure total RNA rapid extraction kit (Beijing Bolingkewei Biotechnology Co. Ltd.). After being quantified by using a Biophotometer (Eppendorf), the same amounts of total RNAs were prepared for synthesizing the first strand cDNAs using PrimeScript ${ }^{\mathrm{TM}}$ RT Reagent Kit (TaKaRa). Then total RNAs of different tissues (cuticle, fat body, hemolymph, Malpighian tubule, midgut, and silk gland) from ten normal larvae of fifth instar were also extracted, respectively, and the cDNA templates were synthesized using the same methods. The relative expression levels of BmLeb5 in hemolymph of the fifth instar larvae at $8,12,16,20,24,30,36,42,48$, and 54 hpi were also detected using qRT-PCR for the two groups. But the expression was detected for the corresponding fat body from 20 hpi because it was difficult to collect the fat body at the early period.

The qRT-PCR reaction system was $20 \mu \mathrm{L}: 10 \mu \mathrm{L} 2 \times$ SYBR Premix Ex Taq ${ }^{\mathrm{TM}}, 0.4 \mu \mathrm{L} 50 \times$ ROX Reference Dye, $0.5 \mu \mathrm{L}$ forward primer LebqF (CGTTTAACCCCAAGCCAATA) and reverse primer LebqR (TGCACTCCGAAATCTTTTGT), and $1 \mu \mathrm{L}$ diluted cDNA template, adding $\mathrm{ddH}_{2} \mathrm{O}$ to $20 \mu \mathrm{L}$. The $\mathrm{qRT}$-PCR reaction program was denaturation at $95^{\circ} \mathrm{C}$ for $5 \mathrm{~min}$, followed by 45 cycles of $95^{\circ} \mathrm{C}$ for $15 \mathrm{~s}, 50^{\circ} \mathrm{C}$ for $15 \mathrm{~s}$, and $72^{\circ} \mathrm{C}$ for $40 \mathrm{~s}$. The $\beta$-actin (NM_001126254.1) was used as internal control by the primers BmactinF (CCGTATGCGAAAGGAAATCA) and BmactinR (TTGGAAGGTAGAGAGGGAGG). The qRT-PCR assays were run on an ABI Prism 7300 Sequence Detection System (Applied Biosystems) according to the protocol of the SYBR Premix Ex Taq Kit (TaKaRa). Each sample was repeated three times. The results were first analyzed with Applied Biosystems 7300 System SDS software RQ Study Application Version 1.4. The relative expression differences of BmLeb5 were calculated by $2^{-\Delta \Delta c t}$ method [34]. Then the results were subjected to IBM SPSS Statistics 19 to analyze the statistical significance. All data were given in terms of relative mRNA expression as terms of means \pm SE.

2.7. Construction of Expression Vectors and Expression of Recombinant BmLeb5. The ORF fragment of BmLeb5 without the predicted signal peptide sequence was amplified using the upstream primer (GGATCCCAGGCTTCGTGCCAGAGG) and the downstream primer (AAGCTTCTATTCTTGGAAAATATC) by gradient PCR. The PCR products were gel-purified and cloned into pMD19-T vector (TaKaRa). After being transformed into E. coli Top 10, the positive clones containing pMD19-T-BmLeb5 were identified by PCR and sequencing. Then the fragment of BmLeb5 from pMD19T-BmLeb5 plasmid was digested with Bam HI and Hind III enzymes, gel-purified, and ligated into the vector $\mathrm{pET}$ $30 \mathrm{a}(+)$ (Novagen). The recombinant expression plasmid pET$30 \mathrm{a}(+)$-BmLeb5 was also confirmed as described above. Then the recombinant plasmid was transformed into the cells of $E$. coli BL21.

The E. coli BL21 with pET-30a(+)-BmLeb5 was cultured overnight (about $12 \mathrm{~h}$ ) in Luria-Bertani (LB) broth containing $30 \mu \mathrm{g} / \mathrm{mL}$ kanamycin. The culture was diluted $1: 100$ into fresh
LB containing $30 \mu \mathrm{g} / \mathrm{mL}$ kanamycin and further incubated at $37^{\circ} \mathrm{C}$ on $220 \mathrm{rpm}$ shaker until $\mathrm{OD}_{600}$ reached 0.6. The isopropyl $\beta$-D-thiogalactoside (IPTG) was added to the culture to a final concentration of $0.2 \mathrm{mM}$ to induce the expression of the recombinant BmLeb5 for $8 \mathrm{~h}$ at $11^{\circ} \mathrm{C}$ or $37^{\circ} \mathrm{C}$. Then the bacterial cells were harvested, respectively, by centrifugation at $12000 \mathrm{~g}$ for $5 \mathrm{~min}$ and resuspended using 2 $\times$ SDS-PAGE loading buffer to analysis the expression by $12 \%$ sodium dodecyl sulfate polyacrylamide gel electrophoresis (SDS-PAGE).

2.8. Purification of BmLeb5. The induced bacterial cells expressing BmLeb5 from the expanding culture at $11^{\circ} \mathrm{C}$ were resuspended in $20 \mathrm{~mL}$ of Binding Buffer $(20 \mathrm{mM}$ Tris- $\mathrm{HCl}$, $0.15 \mathrm{M} \mathrm{NaCl}, \mathrm{pH} 8.0$ ) and subjected to sonication at $400 \mathrm{~W}$ for 20 minutes ( $4 \mathrm{~s}$ working, $8 \mathrm{~s}$ free) on ice. The ultrasonic mixture was centrifuged at $10,000 \mathrm{~g}$ for $20 \mathrm{~min}$. Then the centrifugation supernatant was loaded onto a Ni-IDA-Sepharose column (Novagen) which had been preequilibrated with Binding Buffer. The column was washed using Binding Buffer and Washing Buffer $(20 \mathrm{mM}$ Tris- $\mathrm{HCl}, 20 \mathrm{mM}$ imidazole, and $0.15 \mathrm{M} \mathrm{NaCl}, \mathrm{pH} 8.0)$ until the $\mathrm{OD}_{600}$ of eluate achieved baseline, respectively. The recombinant protein of BmLeb5 was detached by Elution Buffer $(20 \mathrm{mM}$ Tris- $\mathrm{HCl}, 250 \mathrm{mM}$ imidazole, and $0.15 \mathrm{M} \mathrm{NaCl}, \mathrm{pH} 8.0$ ). The last eluate was dialysed by putting into Spectra/Por (R) Universal Closure (Millipore) overnight (about 14h) using phosphate buffer saline (PBS, PH 7.4). The purified protein was detected by $12 \%$ SDS-PAGE. The concentration of purified BmLeb5 was determined by a Bicinchoninic Acid Kit (Beijing Bolingkewei Biotechnology Co. Ltd.).

2.9. Western Blot Analysis. For western blot analysis, $0.1 \mu \mathrm{g}$ of recombinant protein was added in SDS-PAGE gel. Then nonstained SDS-PAGE gel was transferred to polyvinylidene fluoride membrane by electroblotting. After being blocked in PBST $\left(1 \times\right.$ PBS $+0.1 \%$ Tween-20) containing $5 \%\left(\right.$ vol vol $\left.^{-1}\right)$ bovine serum albumin (BSA) for $2 \mathrm{~h}$, the membrane was incubated with mouse anti-His-tag polyclonal antibody in PBST for overnight at $4^{\circ} \mathrm{C}$. Then the membrane was washed three times with PBST and incubated with horseradish peroxidase-conjugated anti-mouse IgG for $1 \mathrm{~h}$, followed by immunoreactivity detection with diaminobenzidine (DAB) as a chromogenic substrate.

2.10. Mass Spectrum Identification. As the molecular weight of recombinant BmLeb5 was bigger than the prediction, Learning Content Management System (LC-MS) was performed to test the recombinant protein molecular weight on TripleTOF ${ }^{\circledR} 6600$ (SCIEX) by Analyst TF 1.7 (AB Sciex) software. After confirmation of the expressed protein containing BmLeb5, Peptide Mass Fingerprinting (PMF) was developed to identify that the peptide sequence was correct using Mascot v2.3 software.

\section{Results and Discussion}

3.1. Confirmation of Fungal Infection. In this study, all the silkworm larvae were infected with cuticle contact 


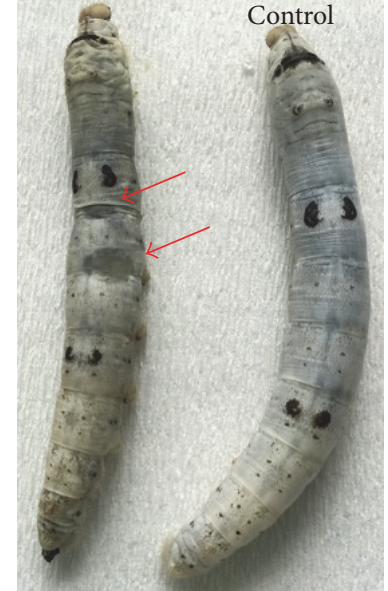

(a)

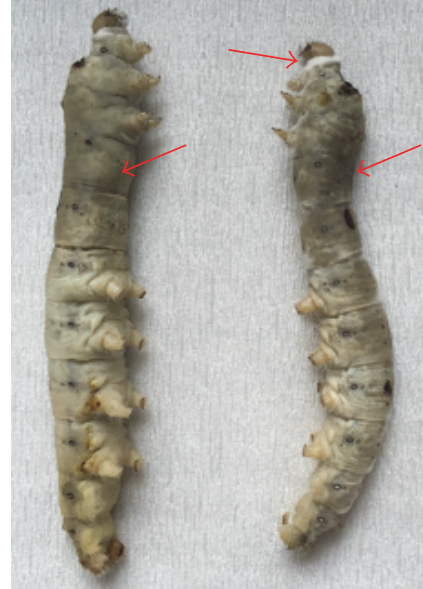

(b)

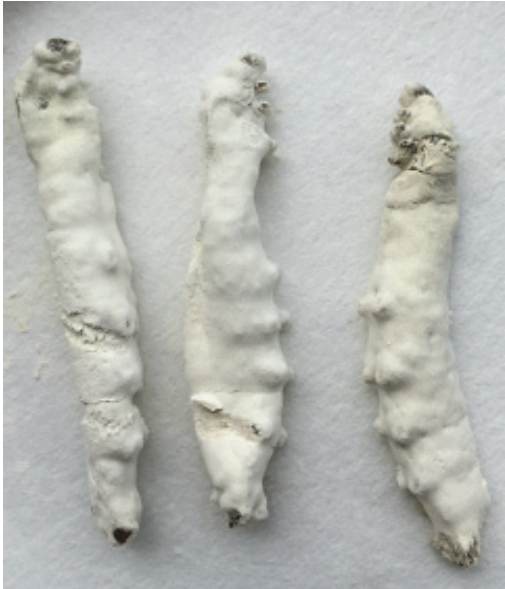

(c)

Figure 1: The disease symptoms of fifth instar silkworm infected by B. bassiana. (a) The oily spots in the body of diseased silkworm larvae. The control silkworms were kept healthy. (b) The large oily spots on the cuticle and the mycelia growing in cuticle junctions. (c) The mycelia and conidia of B. bassiana on dead silkworm body.

inoculation. The successful infection was first confirmed by the appearance of oily spots on the cuticle as typical symptoms and further observation of the blastospores in hemolymph under microscope (Figures 1(a) and 1(b)). For the third instar silkworm, the typical symptoms appeared about $48 \mathrm{hpi}$. The same phenomenon also happened to the fifth instar larvae. Finally, all the inoculated larvae died, and 2 days later the cadavers were all rigid and mycosed (Figure 1(c)), while the larvae of the control groups were kept uninfected and cocooned normally.

3.2. cDNA Cloning and Bioinformatic Analysis. The fulllength cDNA of BmLeb5 was obtained by RACE method according to the manufacturer. After sequencing and assembling, the cDNA of BmLeb5 was deposited in GenBank (Accession number KX100575). It contained 808 bp consisting of a $39 \mathrm{bp} 5^{\prime}$-untranslated region (UTR), a $540 \mathrm{bp}$ ORF, and a 229 bp $3^{\prime}$-UTR (Figure 2). The ORF encodes a proline-rich polypeptide of 179 amino acids with a calculated molecular weight of about $21.1 \mathrm{kDa}$. The isoelectric point was 7.57 at PH 7.0. SignalP 4.1 server predicted that BmLeb5 had a putative $\mathrm{N}$-terminal signal peptide of 20 amino acids, suggesting that it is a secreted protein. Following the signal peptide, there were a putative prosegment of 100 amino acids, a mature peptide of 32 amino acids, and an additional segment of 27 amino acids at the carboxyl terminus (Figure 2). A putative polyadenylation signal of AATAAA was located at 162 nucleotides after translation stop codon and a poly (A) tail was detected in $3^{\prime}$-UTR (Figure 2).

One of the characteristics of $B$. mori lebocins is that they own a unique $\mathrm{O}$-glycosylated threonine residue and the modification seemed to be important for the antimicrobial activity [21,22]. Synthetic lebocins did not show obvious inhibition zones, probably due to their lack of necessary glycosylations $[22,27]$. By NetOGlyc 4.0 Server, an Oglycosylation site was also predicted at $\mathrm{Thr}^{15}$ of BmLeb5 mature peptide as in other peptides of lebocins, suggesting that BmLeb5 is also an O-glycosylated antimicrobial peptide and could also play an important role to defend the microbial invasion (Figure 2) [35].

BmLeb5 is also one of the proline-rich AMPs and owns multiple RXXR motifs which could be recognized by intracellular processing enzymes (Figure 2) [36, 37]. There are four RXXR motifs in the prosegment of BmLeb5: $\mathrm{R}^{39} \mathrm{TVR}^{42}, \mathrm{R}^{83} \mathrm{YVR}^{86}, \mathrm{R}^{117} \mathrm{NTR}^{120}$, and $\mathrm{R}^{151} \mathrm{YRR}^{154}$, following a hydrophilic residue (Figure 2). Most AMPs are synthesized as precursor proteins and then cleaved into some small active peptides to achieve functions. The structure of BmLeb5 implies that the final active peptides of lebocins may be generated from preproteins in the secretory pathway.

Through the BLASTp search, the results of sequence comparison showed that BmLeb5 was $98 \%$ identical to $B$. mori BmLeb3 (NP_001119732.1), 91\% identical to B. mori BmLeb4 (NP_001119731.1), 96\% identical to B. mori BmLeb1/2 (NP_001037468.1), 56\% identical to Antheraea mylitta AmLeb (ABG72704.1) which was the first prorich apidaecin isolated [28], 47\% identical to Pieris rapae PrLeb (AEO21919.1), and 46\% identical to Antheraea pernyi ApLeb (ACB45567.1). So far, 5 lebocin genes were identified in the silkworm. Sequence comparison results implied that these lebocin genes had a very close structural relationship and may be evolved from an ancestral gene. Furthermore, the phylogenetic tree was constructed using the amino acid sequences of BmLeb5 homolog proteins. All the lebocin proteins were derived from lepidoptera insects, implying that lebocins were important AMPs in insects (Figure 3). The phylogenetic relationship of silkworm lebocin family showed that an original ancestral gene first divided into two groups, lebocin 4 gene and a common gene for other 3 genes, and then the latter group was duplicated into lebocin $1 / 2$ and lebocin 3 and 5. Lebocin 5 and 3 owned the highest homology and formed a branch (Figure 3). The discovery of BmLeb5 suggested that the lebocin underwent a further gene evolution in silkworm along with environmental threats. The results also suggested 


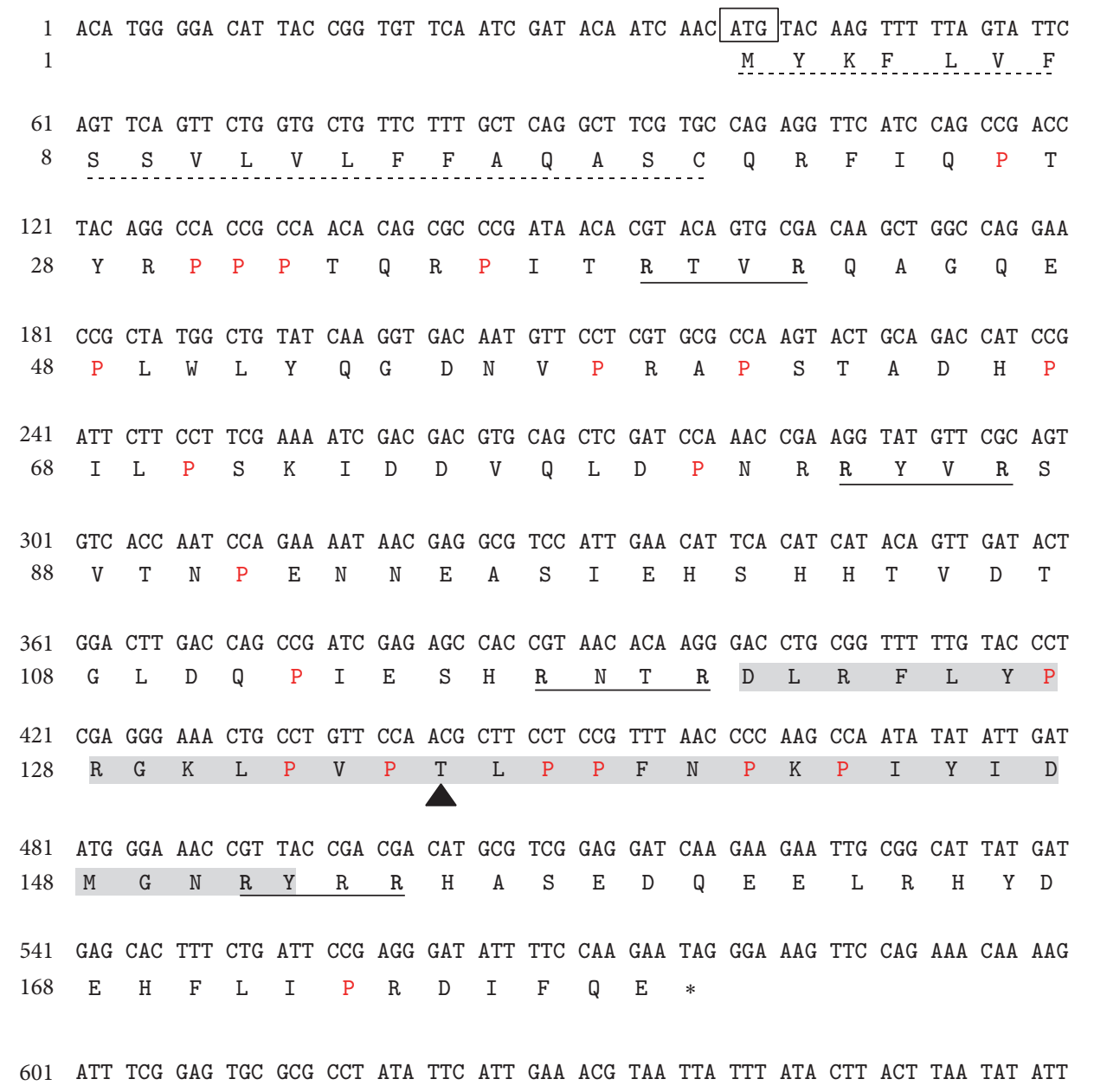

661 TTA TAG TTC TGT TTA AAA ATA CAT AGT AAT TTA TAG TGG CCG TGT CAA ACT CAA ATA ATG

721 TAA GTA CAC ATG TAT AAC ATA ATA ATA TGA AAT TCA ATA AAT CTC AAA ATA TAT TTA TAA

781 AAA AAA AAA AAA AAA AAA AAA AAA AAA A

FIGURE 2: The nucleotide sequence of BmLeb5 full-length cDNA and deduced amino acid sequence. The predicted signal sequence at the $\mathrm{N}$-terminus is marked with a dotted underline. The start codon is boxed. The asterisk $(*)$ represents the stop codon. The polyadenylation signal (AATAAA) is marked with double underlines and polyadenylation tail is underlined. The mat peptide of BmLeb5 is marked in gray bars. The O-glycosylation site is indicated by triangle ( $\mathbf{\Delta})$. The proline-rich polypeptides are marked in red. RXXR motifs are marked in bold arginine and underlined. The numeric positions of the nucleotide and amino acid sequences are shown on the left.

that lebocin owned a multiple gene family as cecropins in the silkworm $[13,15,38]$.

3.3. Expression Profiling of BmLeb5. The relative expression of BmLeb5 was detected by qRT-PCR between the control and infected silkworm larvae. In the third instar whole larvae inoculated with BbHN6, the relative expression of BmLeb5 was upregulated as compared to the normal ones. B. bassiana always could penetrate into silkworm cuticle in $8 \mathrm{hpi}$. So at the early stage of 9 hpi, the expression of BmLeb5 had been upregulated about 5-fold than that in the normal groups.
Afterwards, it was upregulated about 12-, 13-, 33-, 59-, 95-, $62-, 36-$, and 32 -fold than that in control ones at $24,27,30,33$, $36,39,42$, and $45 \mathrm{hpi}$, respectively (Figure $4(\mathrm{a})$ ). The results showed that the expression of BmLeb5 was rapidly induced by $B$. bassiana challenge and reached a maximum difference level of 95 -fold at $36 \mathrm{hpi}$. This was consistent with the results of our previous transcriptome analysis [30]. Then, the tissue distribution of BmLeb5 was further investigated in the fifth instar silkworm larvae with qRT-PCR. The tested tissues included cuticle, fat body, hemolymph, Malpighian tubule, midgut, and silk gland. The BmLeb5 mRNA could be detected 


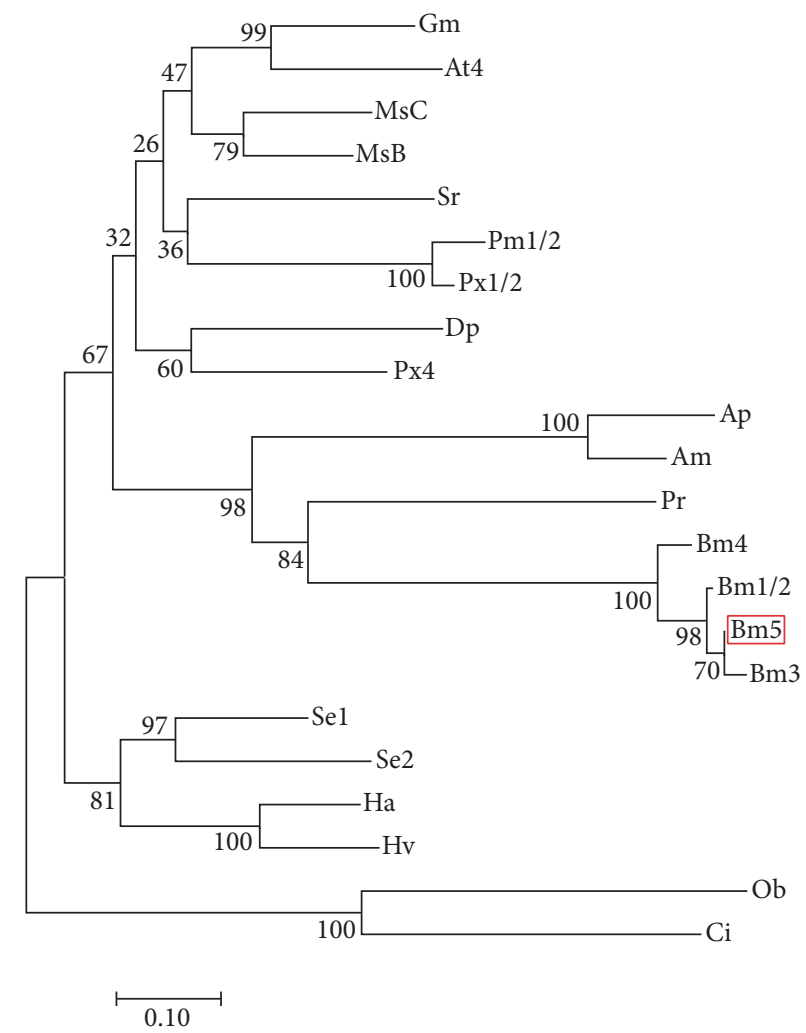

FIGURE 3: Unrooted phylogenetic tree of BmLeb5 and other homologous proteins. The tree was constructed by the neighbor-joining method within the package MEGA 7. Bootstrap majority consensus values on 1,000 replicates are indicated at each branch point (\%). The scale bar represents branch length (number of amino acids substitutions/100 residues). Accession numbers for sequences used in this alignment are as follows (GenBank ID): Bm5 (Bombyx mori, KX100575); Bm3 (Bombyx mori, NP_001119732.1); Bm4 (Bombyx mori, NP_001119731.1); Bm1/2 (Bombyx mori, NP_001037468.1); Sel (Spodoptera exigua, AKJ54499.1); Gm (Galleria mellonella, ACQ99193.1); Ha (Helicoverpa armigera, ALT16900.1); At4 (Amyelois transitella, XP_013188814.1); Ap (Antheraea pernyi, ACB45567.1); MsB (Manduca sexta, ADE20197.1); MsC (Manduca sexta, ADE20198.1); Hv (Heliothis virescens, ACR78447.1); Pm1/2 (Papilio machaon, KPJ06168.1); Px1/2 (Papilio xuthus, KPI91575.1); Se2 (Spodoptera exigua, AKJ54500.1); Sr (Samia ricini, BAD84189.1); Dp (Danaus plexippus, EHJ64534.1); Px4 (Papilio xuthus, KPI91576.1); Pr (Pieris rapae, AEO21919.1); Am (Antheraea mylitta, ABG72704.1); Ob (Operophtera brumata, KOB78261.1); Ci (Chrysodeixis includens, AAS48093.1).

in all the tested tissues of normal larvae but the highest in the fat body (Figure 4(b)). While the relative expressions of $B m L e b 5$ were only upregulated in fat body and hemolymph in the inoculated larvae, no obvious changes were detected in the other four tissues. The maximum upregulation of expression was approximately 20 -fold in fat body as compared to the normal ones (Figure 4(c)). Significant difference in hemolymph achieved 22 -fold at 20 hpi (Figure $4(\mathrm{~d})$ ). When the internal control of $\beta$-actin was changed to GAPDH, the general trend of expression changes was the same.

Previous studies had demonstrated that $B$. mori lebocin $1 / 2$ could be induced and strongly expressed in fat body by bacterial injection [20]. B. mori lebocin 3 and lebocin 4 were showed to be induced in the fat body and hemocytes by lipopolysaccharide (LPS) [21]. Our qRT-PCR results for lebocin 5 were consistent with these reports. Without exception, the lebocins are expressed the highest in fat body of silkworm. B. mori lebocin 1-3 could be induced by E. coli and $B$. mori lebocin 4 could be induced by LPS $[20,22,29]$. Furthermore, the purified peptides of lebocin 1-3 showed antibacterial activity against some Gram-negative bacteria
[22]. So far, there are no reports indicating that fungi could induce B. mori lebocin expression. Our results suggested that BmLeb5 not only could be induced to the upregulated expression by fungi, but also maybe play an important role in the silkworm to defend B. bassiana infection.

3.4. Expression and Purification of Recombinant BmLeb5. The recombinant expression vector pET-30a(+)-BmLeb5 was constructed by inserting the BmLeb5 ORF fragment (without signal peptide sequence) and transformed into E. coli BL21 for expression of BmLeb5. After IPTG induction at $11^{\circ} \mathrm{C}$ and $37^{\circ} \mathrm{C}$, respectively, the expression quantity of fusion protein was detected by SDS-PAGE. Though the expression was commonly induced at the temperature of $37^{\circ} \mathrm{C}$, the induced expression efficiency for BmLeb5 at $11^{\circ} \mathrm{C}$ was significantly higher than at $37^{\circ} \mathrm{C}$ (Figure 5(a)). As we know, there are no reports about the successful in vitro expression of any $B$. mori lebocin genes. In Manduca sexta, only fusion protein of lebocin B was expressed at $16^{\circ} \mathrm{C}$ after IPTG induction [27].

After being purified using a Ni-IDA-Sepharose column, the recombinant BmLeb5 exhibited a significant 

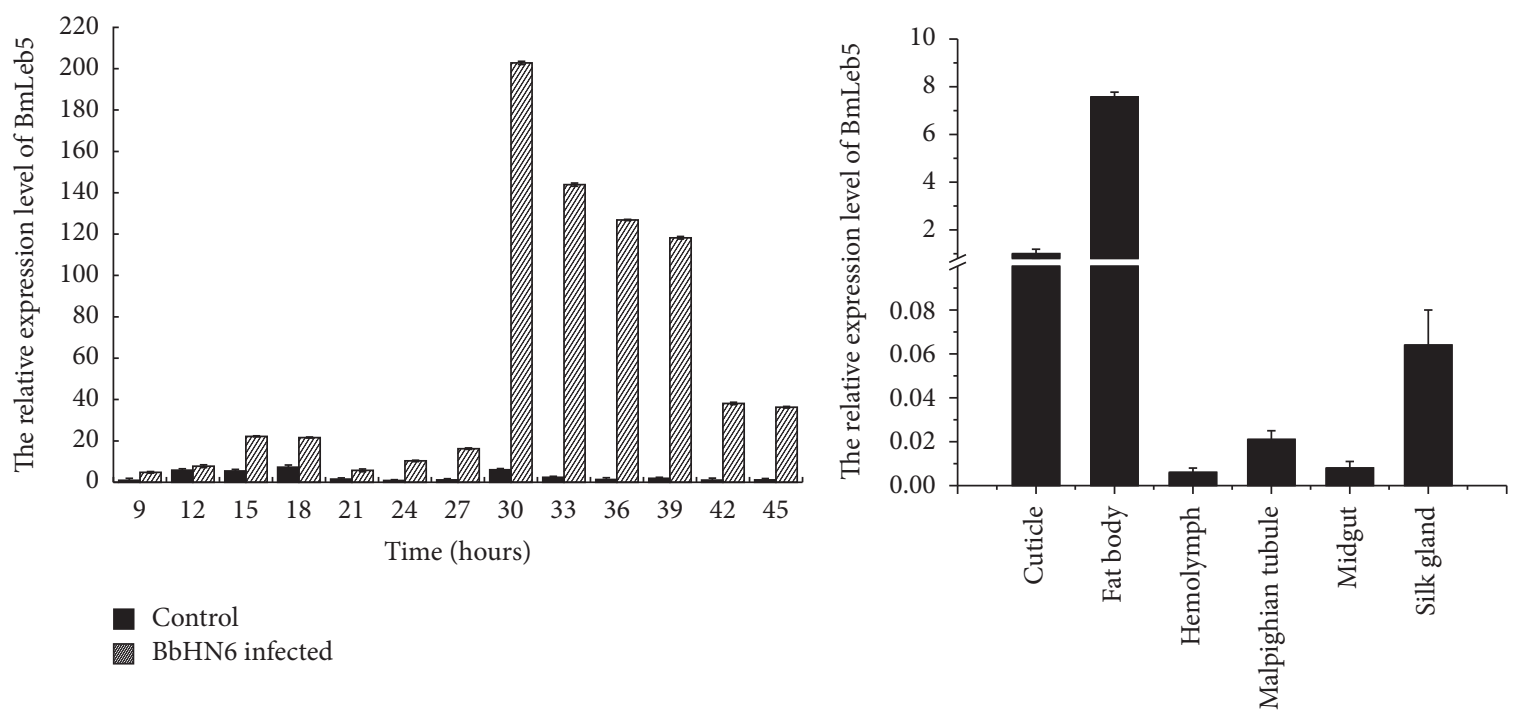

(a)

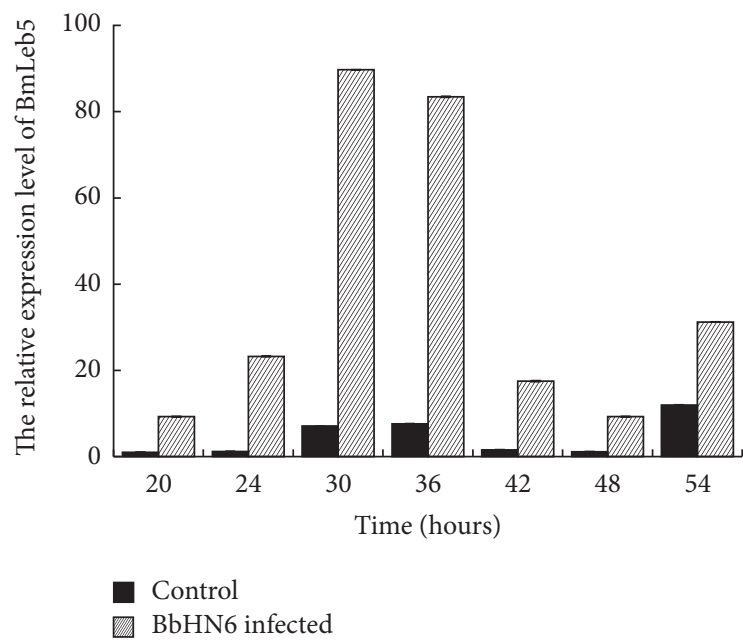

(c) (b)

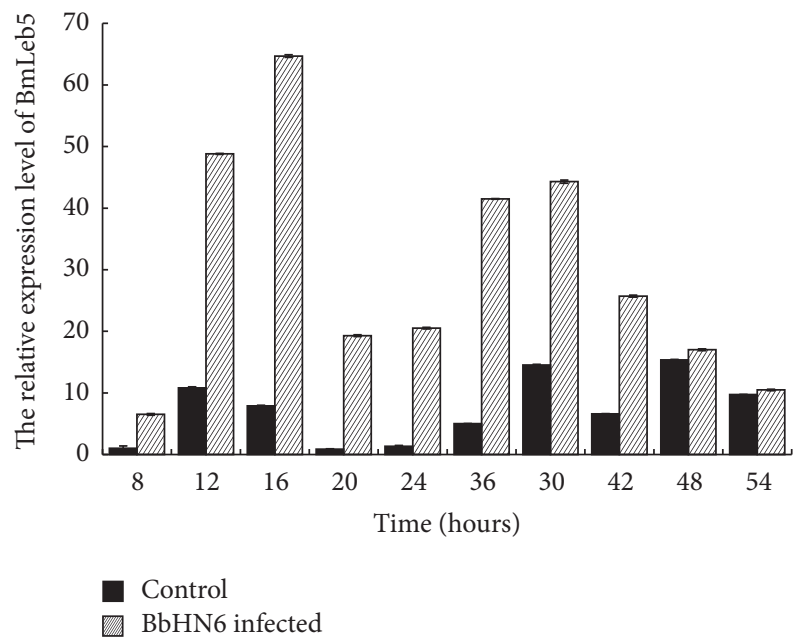

(d)

FIGURE 4: The relative mRNA expression levels of BmLeb5. (a) In the third instar whole larvae of BbHN6-infected and control groups at different times. (b) In different tissues of normal fifth instar larvae. (c) In the fat body of BbHN6-infected and control fifth instar larvae at different times. (d) In the hemolymph of BbHN6-infected and control fifth instar larvae at different times. The $y$-axis indicates the relative expression level of BmLeb5 mRNA transcripts. Vertical bars represent the mean $\pm \mathrm{SE}(n=3)$.

fusion protein band of approximately $32 \mathrm{kDa}$ on $12 \%$ SDS-PAGE followed by staining with Coomassie Brilliant Blue R-250. Western blot assay was used to further confirm the purified recombinant protein BmLeb5 using antihistidine antibody. As shown in Figure 5(b), the single protein band revealed that the prokaryotic expression system achieved an excellent inducible expression of BmLeb5. The expressed recombinant protein would be used for the detection of antifungal activity of BmLeb5 and its antifungal mechanism.

3.5. Confirmation of the Recombinant Protein of BmLeb5. By SDS-PAGE and western blot analysis, the molecular mass of recombinant $\mathrm{BmLeb} 5$ was approximately $32 \mathrm{kDa}$, higher than the predicted $24.6 \mathrm{kDa}$. In order to confirm the target protein, LC-MS was used to detect the size of molecular mass of the purified recombinant protein. Two peaks of molecular mass, corresponding to $24.6 \mathrm{kDa}$ and $31.8 \mathrm{kDa}$ (Figure 6), were presented. The result could judge that the two substances of $24.6 \mathrm{kDa}$ and $31.8 \mathrm{kDa}$ were main ingredients in the solution of recombinant protein. The $24.6 \mathrm{kDa}$ peak was consistent with the predicted results. After trypsin enzymolysis and secondary mass spectrum identification, the sequences of peptide fragments were blasted with database of UniProt by Mascot v2.3 software. 12 peptides were consistent with the sequence of BmLeb5. After assembling, the vast majority of fragments were completely in accordance with the recombinant protein of BmLeb5. The other peptide of $31.8 \mathrm{kDa}$ was blasted as a protein kinase which probably was reassembled from serine residues of the added tryptase in the solution. Therefore, we established successfully for the first time the expression system for BmLeb5 and the expressed protein 


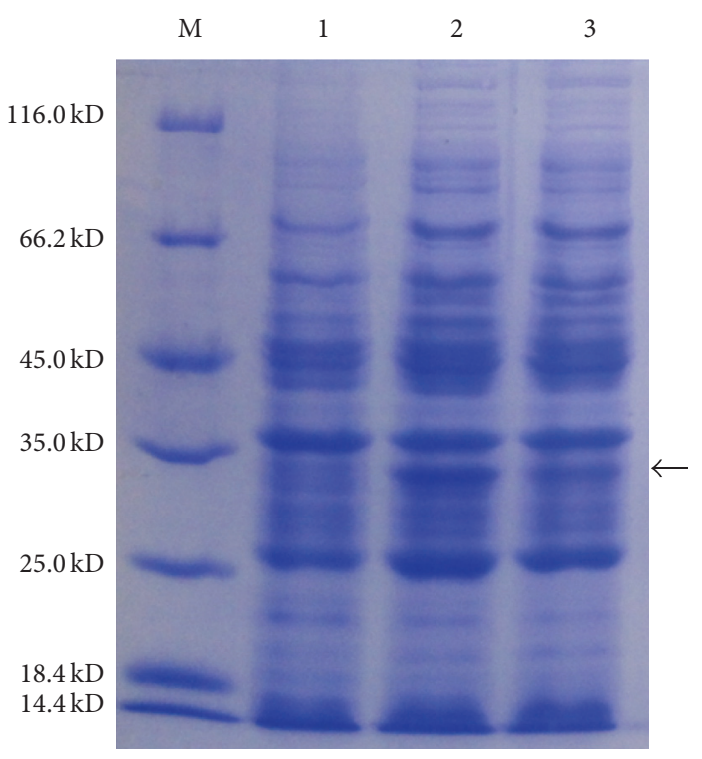

(a)

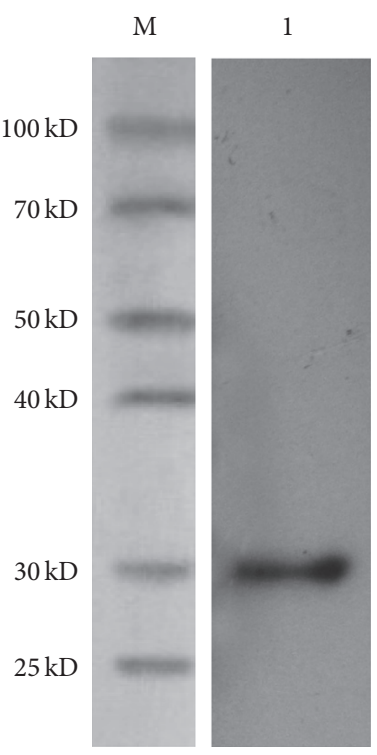

(b)

FIGURE 5: SDS-PAGE and western blot analysis of recombinant BmLeb5. (a) SDS-PAGE analysis of recombinant BmLeb5 expressed at different induction temperatures. Lane M: protein marker (14.4-116 kDa); lane 1: uninduced; lane 2: induced at $11^{\circ} \mathrm{C}$; lane 3 : induced at $37^{\circ} \mathrm{C}$. $(\mathrm{b}) \mathrm{Western}$ blot analysis of purified recombinant BmLeb5. Lane M: protein marker (25-100 kDa); lane 1: $0.1 \mu \mathrm{g}$ recombinant protein of BmLeb5.

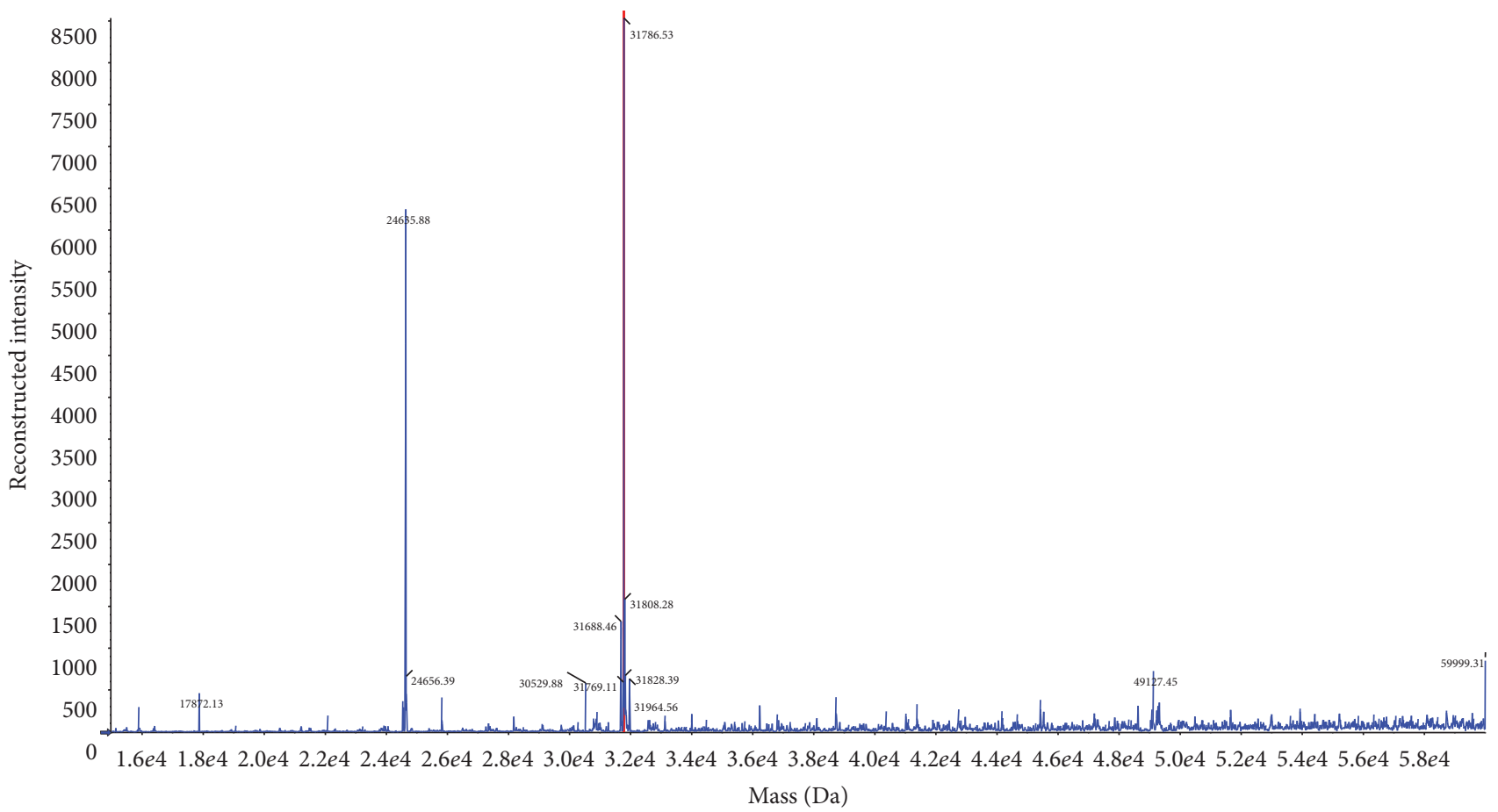

FIGURE 6: Quality analysis of purified recombinant BmLeb5 with mass spectrometry. There were two substances of $24.6 \mathrm{kDa}$ and $31.8 \mathrm{kDa}$ in the solution of recombinant protein. The $24.6 \mathrm{kDa}$ peak was consistent with the predicted result.

could be used for further study of the roles of lebocins both in vitro and in vivo in the silkworm.

In conclusion, we successfully cloned the full-length cDNA of BmLeb5 gene from silkworm using RACE and the sequence was submitted to GenBank. The deduced amino acid sequence contains an O-glycosylation site and four
RXXR motifs. The phylogenetic tree suggests that lebocins form a multiple gene family in silkworm. The upregulated expression of BmLeb5 in the whole silkworm larvae, in the fat body, and in hemolymph indicates that it might play an important role in the immune response of silkworm to defend the $B$. bassiana infection. The recombinant protein 
of BmLeb5 was for the first time successfully expressed with prokaryotic expression system and then purified with $\mathrm{Ni}$ IDA-Sepharose column. We also confirmed the molecular mass and sequences of the expressed protein by LC-MS and PMF. Details of antifungal mechanism of the BmLeb5 in vivo remain to be explored.

\section{Competing Interests}

The authors declare there is no conflict of interests.

\section{Acknowledgments}

This work was supported by the National Natural Science Foundation of China (Grant no. 31272498), the Specialized Research Fund for the Doctoral Program of Higher Education of China (Grant no. 20123220110003), the Graduate Science and Technology Innovation Plan of Jiangsu Province (Grant no. KYZZ16_0205), and the Natural Science Foundation of Jiangsu Province (Grant no. BK20161364).

\section{References}

[1] C.-X. Hou, G.-X. Qin, T. Liu et al., "Differentially expressed genes in the cuticle and hemolymph of the silkworm, Bombyx mori, injected with the fungus Beauveria bassiana," Journal of Insect Science, vol. 13, article 138, 14 pages, 2013.

[2] X. Chen, C. Huang, L. He, S. Zhang, and Z. Li, "Molecular tracing of white muscardine in the silkworm, Bombyx mori (Linn.) II. Silkworm white muscardine is not caused by artificial release or natural epizootic of Beauveria bassiana in China," Journal of Invertebrate Pathology, vol. 125, pp. 16-22, 2015.

[3] E. A. Steinhaus, "Microbial diseases of insects," Annual review of microbiology, vol. 11, pp. 165-182, 1957.

[4] D. Lu, M. Pava-Ripoll, Z. Li, and C. Wang, "Insecticidal evaluation of Beauveria bassiana engineered to express a scorpion neurotoxin and a cuticle degrading protease," Applied Microbiology and Biotechnology, vol. 81, no. 3, pp. 515-522, 2008.

[5] M. D. Lavine and M. R. Strand, "Insect hemocytes and their role in immunity," Insect Biochemistry and Molecular Biology, vol. 32, no. 10, pp. 1295-1309, 2002.

[6] N. A. Ratcliffe, "Invertebrate immunity-a primer for the nonspecialist," Immunology Letters, vol. 10, no. 5, pp. 253-270, 1985.

[7] P. Bulet, R. Stöcklin, and L. Menin, "Anti-microbial peptides: from invertebrates to vertebrates," Immunological Reviews, vol. 198, no. 1, pp. 169-184, 2004.

[8] C. Luna, X. Wang, Y. Huang, J. Zhang, and L. Zheng, "Characterization of four Toll related genes during development and immune responses in Anopheles gambiae," Insect Biochemistry and Molecular Biology, vol. 32, no. 9, pp. 1171-1179, 2002.

[9] P. Bulet, C. Hetru, J.-L. Dimarcq, and D. Hoffmann, "Antimicrobial peptides in insects; structure and function," Developmental and Comparative Immunology, vol. 23, no. 4-5, pp. 329-344, 1999.

[10] B. Lemaitre and J. Hoffmann, "The host defense of Drosophila melanogaster," Annual Review of Immunology, vol. 25, no. 1, pp. 697-743, 2007.

[11] C. Hetru and J. A. Hoffmann, "NF-kappaB in the immune response of Drosophila," Cold Spring Harbor perspectives in biology, vol. 1, no. 6, p. a000232, 2009.
[12] T. Cheng, P. Zhao, C. Liu et al., "Structures, regulatory regions, and inductive expression patterns of antimicrobial peptide genes in the silkworm Bombyx mori," Genomics, vol. 87, no. 3, pp. 356-365, 2006.

[13] Y. Yamano, M. Matsumoto, K. Sasahara, E. Sakamoto, and I. Morishima, "Structure of genes for cecropin A and an inducible nuclear protein that binds to the promoter region of the genes from the silkworm, Bombyx mori," Bioscience, Biotechnology and Biochemistry, vol. 62, no. 2, pp. 237-241, 1998.

[14] K. Taniai, K. Kadono-Okuda, Y. Kato et al., "Structure of two cecropin B-encoding genes and bacteria-inducible DNAbinding proteins which bind to the $5 /$-upstream regulatory region in the silkworm, Bombyx mori," Gene, vol. 163, no. 2, pp. 215-219, 1995.

[15] J. Yang, S. Furukawa, A. Sagisaka et al., "cDNA cloning and gene expression of cecropin $\mathrm{D}$, an antibacterial protein in the silkworm, Bombyx mori," Comparative Biochemistry and Physiology -B Biochemistry and Molecular Biology, vol. 122, no. 4, pp. 409-414, 1999.

[16] S. Hara and M. Yamakawa, "Moricin, a novel type of antibacterial peptide isolated from the silkworm, Bombyx mori," Journal of Biological Chemistry, vol. 270, no. 50, pp. 29923-29927, 1995.

[17] S. Kawaoka, S. Katsuma, T. Daimon et al., "Functional analysis of four gloverin-like genes in the silkworm, Bombyx mori," Archives of Insect Biochemistry and Physiology, vol. 67, no. 2, pp. 87-96, 2008.

[18] M. Sugiyama, H. Kuniyoshi, E. Kotani et al., "Characterization of a Bombyx moricDNA encoding a novel member of the attacin family of insect antibacterial proteins," Insect Biochemistry and Molecular Biology, vol. 25, no. 3, pp. 385-392, 1995.

[19] S. H. Kim, B. S. Park, E. Y. Yun et al., "Cloning and expression of a novel gene encoding a new antibacterial peptide from silkworm, Bombyx mori," Biochemical and Biophysical Research Communications, vol. 246, no. 2, pp. 388-392, 1998.

[20] S. Chowdhury, K. Taniai, S. Hara et al., "cDNA cloning and gene expression of lebocin, a novel member of antibacterial peptides from the silkworm, Bombyx mori," Biochemical and Biophysical Research Communications, vol. 214, no. 1, pp. 271-278, 1995.

[21] S. Furukawa, K. Taniai, J. Ishibashi, S. Hara, T. Shono, and M. Yamakawa, "A novel member of lebocin gene family from the silkworm, Bombyx mori," Biochemical and Biophysical Research Communications, vol. 238, no. 3, pp. 769-774, 1997.

[22] S. Hara and M. Yamakawa, "A novel antibacterial peptide family isolated from the silkworm, Bombyx mori," Biochemical Journal, vol. 310, no. 2, pp. 651-656, 1995.

[23] G. Liu, D. Kang, and H. Steiner, "Trichoplusia ni lebocin, an inducible immune gene with a downstream insertion element," Biochemical and Biophysical Research Communications, vol. 269, no. 3, pp. 803-807, 2000.

[24] Y. Bao, Y. Yamano, and I. Morishima, "A novel lebocin-like gene from eri-silkworm, Samia cynthia ricini, that does not encode the antibacterial peptide lebocin," Comparative Biochemistry and Physiology - B Biochemistry and Molecular Biology, vol. 140, no. 1, pp. 127-131, 2005.

[25] M. D. Lavine, G. Chen, and M. R. Strand, "Immune challenge differentially affects transcript abundance of three antimicrobial peptides in hemocytes from the moth Pseudoplusia includens," Insect Biochemistry and Molecular Biology, vol. 35, no. 12, pp. 1335-1346, 2005.

[26] S. Rayaprolu, Y. Wang, M. R. Kanost, S. Hartson, and H. Jiang, "Functional analysis of four processing products from multiple 
precursors encoded by a lebocin-related gene from Manduca sexta," Developmental and Comparative Immunology, vol. 34, no. 6, pp. 638-647, 2010.

[27] X.-J. Rao, X.-X. Xu, and X.-Q. Yu, "Functional analysis of two lebocin-related proteins from Manduca sexta," Insect Biochemistry and Molecular Biology, vol. 42, no. 4, pp. 231-239, 2012.

[28] K. Casteels-Josson, W. Zhang, T. Capaci, P. Casteels, and P. Tempst, "Acute transcriptional response of the honeybee peptide-antibiotics gene repertoire and required posttranslational conversion of the precursor structures," Journal of Biological Chemistry, vol. 269, no. 46, pp. 28569-28575, 1994.

[29] S. Y. Hara and M. Yamakawa, "Cooperative antibacterial relationship between lebocin and cecropin $\mathrm{D}$, antibacterial peptides isolated from the silkworm, Bombyx mori (Lepidoptera: Bombycidae)," Applied Entomology and Zoology, vol. 30, no. 4, pp. 606-608, 1995.

[30] C. Hou, G. Qin, T. Liu et al., “Transcriptome analysis of silkworm, Bombyx mori, during early response to Beauveria bassiana challenges," PLoS ONE, vol. 9, no. 3, Article ID e91189, 2014.

[31] D. Lu, T. Geng, C. Hou, Y. Huang, G. Qin, and X. Guo, "Bombyx mori cecropin A has a high antifungal activity to entomopathogenic fungus Beauveria bassiana," Gene, vol. 583, no. 1, pp. 29-35, 2016.

[32] S. Kumar, G. Stecher, and K. Tamura, "MEGA7: molecular evolutionary genetics analysis version 7.0 for bigger datasets," Molecular Biology and Evolution, vol. 33, no. 7, pp. 1870-1874, 2016.

[33] N. Saitou and M. Nei, "The neighbor-joining method: a new method for reconstructing phylogenetic trees," Molecular Biology and Evolution, vol. 4, no. 4, pp. 406-425, 1987.

[34] K. J. Livak and T. D. Schmittgen, "Analysis of relative gene expression data using real-time quantitative PCR and the 2$\Delta \Delta$ CT method," Methods, vol. 25, no. 4, pp. 402-408, 2001.

[35] I. B. H. Wilson, Y. Gavel, and G. Von Heijne, "Amino acid distributions around O-linked glycosylation sites," Biochemical Journal, vol. 275, no. 2, pp. 529-534, 1991.

[36] L. Devi, "Consensus sequence for processing of peptide precursors at monobasic sites," FEBS Letters, vol. 280, no. 2, pp. 189194, 1991.

[37] J. A. Veenstra, "Mono- and dibasic proteolytic cleavage sites in insect neuroendocrine peptide precursors," Archives of Insect Biochemistry and Physiology, vol. 43, no. 2, pp. 49-63, 2000.

[38] Y. Yamano, M. Matsumoto, K. Inoue, T. Kawabata, and I. Morishima, "Cloning of cdnas for cecropins a and b, and expression of the genes in the silkworm, bombyx mori," Bioscience, Biotechnology and Biochemistry, vol. 58, no. 8, pp. 1476-1478, 1994. 

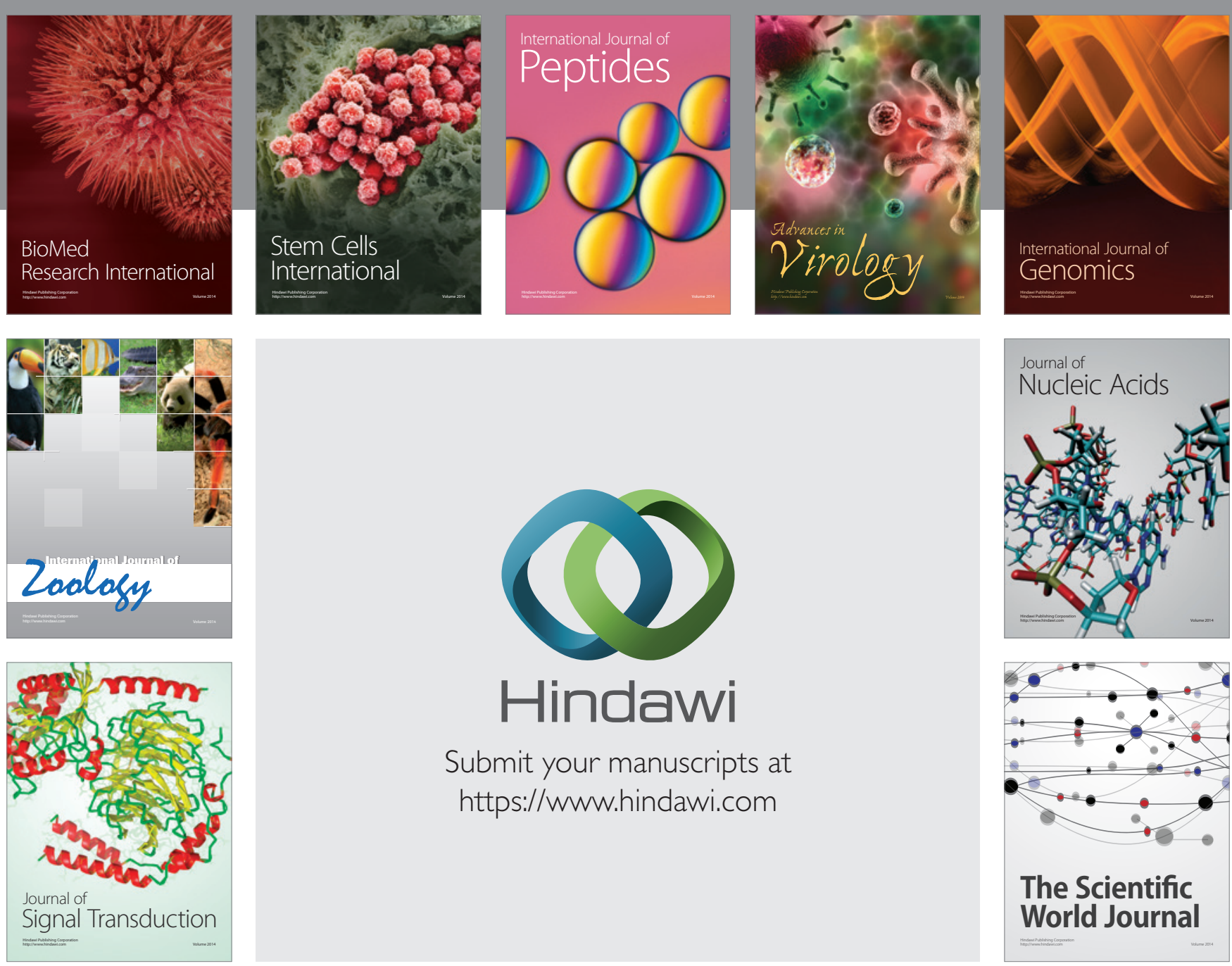

Submit your manuscripts at

https://www.hindawi.com
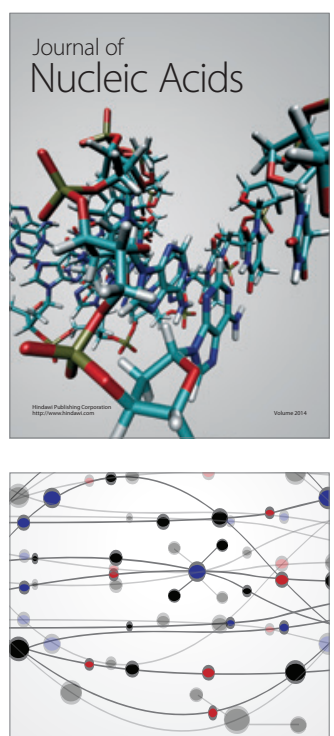

The Scientific World Journal
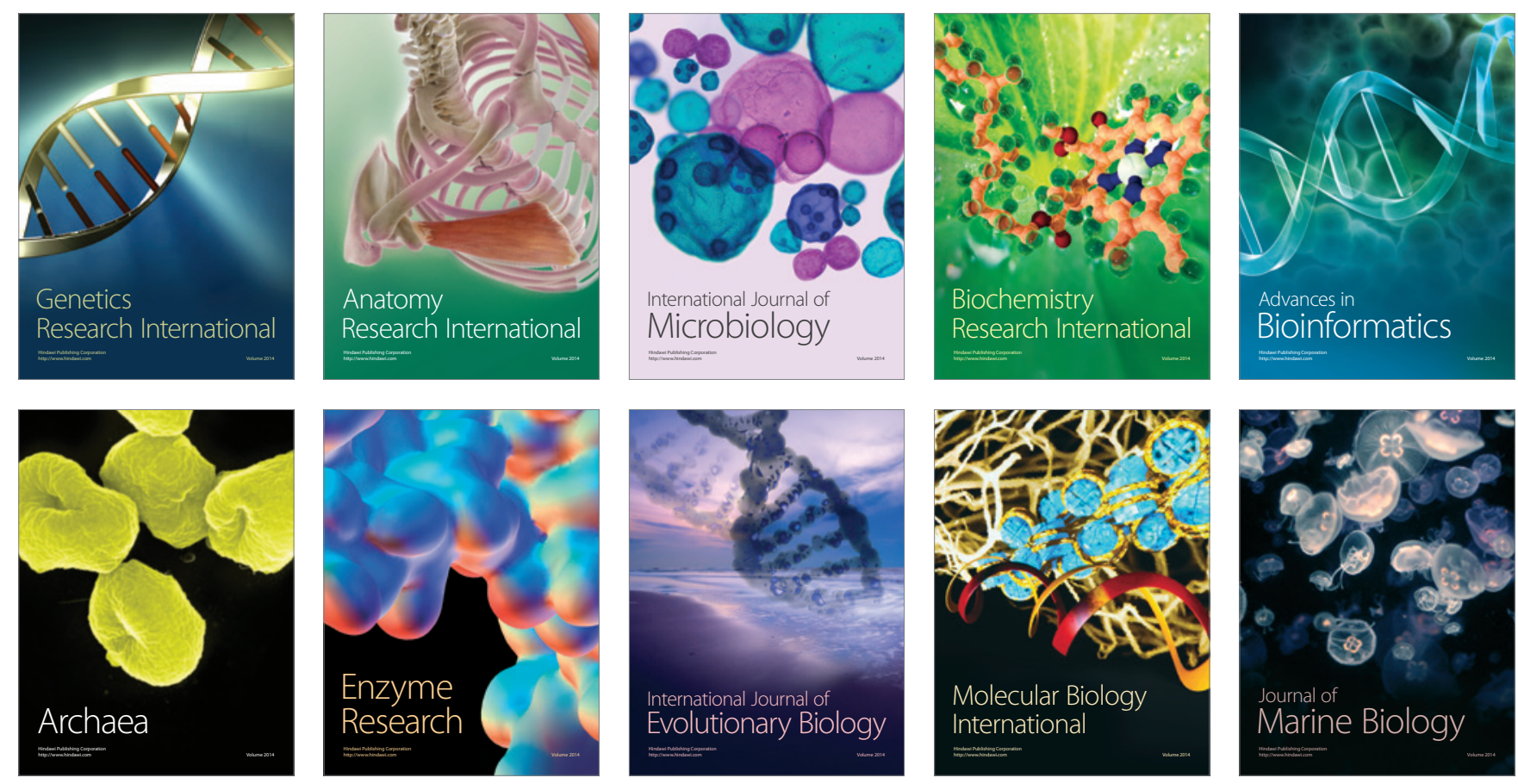